\title{
Dynamic analysis and structure optimization of a floating ring system in dry gas seal
}

\author{
Yun LIU*, Quanxing LIU**, Ming YIN*** and Guofu YIN* \\ *School of Aeronautics and Astronautics, Sichuan University, Chengdu, 610065, P.R. China \\ E-mail: gfyin@scu.edu.cn \\ **Sichuan Aerospace Vocational College, Chengdu, 610100, P.R. China \\ ${ }^{* * *}$ School of Manufacturing Science and Engineering, Sichuan University, Chengdu, 610065, P.R. China
}

Received: 27 April 2018; Revised: 24 August 2018; Accepted: 28 November 2018

\begin{abstract}
Dry gas seal (DGS) is an important way for the nuclear reaction seal and the dynamic property of floating ring system (FRS) is the key to the reliability of DGS. In this study, the vibration characteristics of FRS were obtained through the test experiment under the designed multi-stage discretization operation conditions. A new dynamic model of FRS was established based on the finite element method. The dynamic analysis of FRS was achieved by the means of the pre-stressed modal analysis (PSMA). Based on the spectrum analysis of the experimental data, the modal identification was performed by peak picking method, which verified the dynamic analysis result. The results show that the dynamic performance of the FRS was not sufficient to achieve the absolute seal reliability required for nuclear reactions. An integrated structure optimization method based on the PSMA, response surface methodology (RSM) and particle swarm optimization (PSO) was used to optimize the dynamic property of FRS. The RSM and the Box-Behnken (BB) experiment design were combined to construct the complete quadratic polynomial response surface (RS) models of the natural frequency and static deformation in terms of between the optimized variables, including the uniform spring stiffness and three main axial structure sizes. Under the condition that the static deformation was less than the expected value and the natural frequency value was set as the objective function, the natural frequency was increased by $15 \%$ through the velocity- position model of PSO. It was indicated that the dynamic property of FRS was significantly improved. The dynamic analysis of FRS enriches the dynamic characteristics study of the whole DGS system and the integrated optimization method achieves the parameters optimization of DGS system applied to the nuclear reaction seal.
\end{abstract}

Keywords : Floating ring system, Multi-stage discretization, Operation conditions, Pre-stressed modal analysis, Response surface methodology, Particle swarm optimization, Integrated optimization

\section{Introduction}

Dry gas seals (DGS) is a multi-component device as shown in Fig. 1. The DGS is designed to prevent gas from escaping from one region to another through a gas film between the rotating and floating mating rings. The floating ring is flexibly mounted and the rotating ring is rigidly mounted [Miller et al., 2002]. With the advantages of wear resistance and low pollution, the DGS is widely used in the nuclear power fields [Faria, 2001]. The seals of nuclear power require absolute reliability, especially under limit conditions (high medium pressure and high rotational speed) [Wang et al., 2011]. The dynamic characteristics studies of the gas film, rotating ring system and floating ring system (FRS) is of great importance for the seal reliability.

A considerable body of work has been published concerning the dynamic characteristics of gas film to improve the seal reliability. Zhou and Gu [2008] analyzed the influence of periodic axial micro-disturbance on the thickness and bearing capacity of gas film. Considering the external shock disturbances, Lee and Zheng [2013] accomplished the 
analysis of both steady performance and dynamic tracking of gas film through the direct dynamic analysis and finite element analysis. The research on the rotating ring system also has aroused much interest committed to the improvement of seal reliability. Jiao et al. [2016] used the orthogonal experimental design to collect the experimental data and analyzed the experimental data on process parameters to determine the optimal conditions of the rotating ring groove process. Li et al. [2011] established a dynamic model of the rotating system based on the Hamilton principle and the finite element method to analyze the dynamic behavior of the rotating system with the coupled effects of the film force, the seal force, and the mass eccentricity of the disk.

Compared with the dynamic analysis of gas film and rotating ring system, the research on the FRS (floating ring, thrusting ring and spring seat) is less. The floating ring is flexibly mounted to the spring seat with a spring. when designed properly, the FRS can be beneficial, but it can also be detrimental if the system is designed improperly. An improperly design is that the system dynamic property contributes to a natural resonance close to an operating frequency. When the rated medium pressure or rated rotational speed is certain value, there is a risk that the floating ring will have an excessive axial movement leading to the seal unreliability. Clearly, the dynamic property of FRS is critically important to the successful function and performance of the DGS. In this work, the research involves the whole FRS [Wang et al., 2013]. According to the analytical expression of gas film stiffness and the vibration equation of FRS, the vibration characteristics of FRS is related to the stability of the gas film. For the dynamic analysis of the FRS, an experiment platform based on LMS Test Lab Measurement System for the dynamic characteristics of FRS was set up and the leakage reflecting the state of gas film was monitored. We designed the multi-stage discretization operation conditions to obtain the axial vibration acceleration responses of FRS and the leakage values. The change of the vibration acceleration response curves and the leakage curves verified that the dynamic characteristics of FRS affected the stability of the gas film. The peak of the curves proved that the system resonance and the instability of gas film had happened. To overcome the unreliability of DGS, an efficient integrated optimization method based on the pre-stressed modal analysis (PSMA), response surface methodology (RSM) and particle swarm optimization (PSO) was proposed to optimize the structure of the FRS. Reference to the practical design of the DGS used for nuclear reactions, the PSMA of the whole FRS was completed in details. The PSMA obtained the system natural frequency and the static deformation simultaneously. The results of spectrum analysis through the vibration acceleration response certified the model accuracy of PSMA. Compared with the orthogonal experiment design and the central composite design, the Box-Behnken (BB) experiment design can get accurate response model with simple design [Kittidecha and Marasinghe, 2015]. The RSM and the BB experiment design were combined to construct the complete quadratic polynomial response surface (RS) models. The relations between the optimization variables and the natural frequency and static deformation were obtained using the RS models. Tan et al. [2017] and Xie et al. [2009] optimized their structures using the genetic algorithm, which is suitable for the optimization with multivariable, especially above 10 variables. However, due to the DGS involving nesting design, the circumferential and radial dimensions cannot be changed. There were 4 axial parameters of the FRS selected as the optimization variables in this paper. The unique velocity- position model of PSO algorithm is simple and avoid the complex genetic operations, which is suitable for the optimization of the 4 axial parameters. Setting the FRS natural frequency as the objective function and the static deformation as the constraint in PSO, the natural frequency had been significantly improved with the static deformation less than the expected value. The dynamic analysis of FRS enriches the dynamic characteristics study of the whole DGS system. The integrated optimization method based on PSMA, RSM and PSO provides an efficient means for the dynamic properties optimization of the DGS applied to nuclear reaction seal.

\section{The principle of dynamic characteristics analysis}

When the rotating ring is working, the floating ring can flexibly and periodically move along the axial direction. The sealing gas film is between the rotating ring and the floating ring. The stable axial stiffness of sealing gas film ensures the low leakage and the reliability of sealing performance. The analytical expression of gas film stiffness $K$ is [Ding et al., 2011]

$$
K=\frac{\mathrm{d} F}{\mathrm{~d} \delta} .
$$

Where $F$ denotes the gas film open force and $\delta$ denotes the gas film thickness 


$$
\begin{aligned}
& F=\left[\frac{p_{i}}{1-\Delta h \cos \phi-\left(\frac{E \cos \omega}{E+\delta}\right)}+\frac{\frac{E p_{i}}{E+\delta}\left(\eta_{1(\zeta)} \cos \omega+\eta_{2(\zeta)} \cos \omega\right)}{1-\Delta h \cos \phi-\left(\frac{E \cos \omega}{E+\delta}\right)}-\frac{3}{2} \beta_{0}\left(\frac{E}{E+\delta}\right)^{2} \eta_{2(\zeta)}\left(\zeta_{0}-\zeta\right) p_{i}\right] \times\left(\pi R_{o}^{2}-\pi R_{i}^{2}\right) \\
& \eta_{1(\zeta)}=c_{10} \exp \left(\sqrt{\beta_{1}} \zeta\right)+c_{10} \exp \left(-\sqrt{\beta_{1}} \zeta\right)+\left[c_{11} \exp \left(\sqrt{\beta_{1}} \zeta\right)+c_{11} \exp \left(-\sqrt{\beta_{1}} \zeta\right)-\frac{A_{1}}{2 \sqrt{\beta_{1}}} \zeta \exp \left(\sqrt{\beta_{1}} \zeta\right)-\frac{B_{1}}{2 \sqrt{\beta_{1}}} \zeta \exp \left(-\sqrt{\beta_{1}} \zeta\right)\right] \varepsilon \\
& \eta_{2(\zeta)}=c_{20} \exp \left(\sqrt{\beta_{1}} \zeta\right)+c_{20} \exp \left(-\sqrt{\beta_{1}} \zeta\right)+\left[c_{21} \exp \left(\sqrt{\beta_{1}} \zeta\right)+c_{21} \exp \left(-\sqrt{\beta_{1}} \zeta\right) \frac{A_{2}}{2 \sqrt{\beta_{1}}} \zeta \exp \left(\sqrt{\beta_{1}} \zeta\right)-\frac{B_{2}}{2 \sqrt{\beta_{1}}} \zeta \exp \left(-\beta_{1} \zeta\right)-\frac{\alpha_{2}}{\beta_{1}}\right] \varepsilon
\end{aligned}
$$

Where $\phi=\frac{x}{R_{i}}$ denotes the dimensionless polar angle; $\zeta_{0}=\frac{R_{o}}{R_{i}}$ denotes the dimensionless outside diameter; $\zeta=\frac{y}{R_{i}}$ denotes the dimensionless polar radius; $R_{o}$ denotes the outside diameter of rotating ring; $R_{i}$ denotes the inner diameter of rotating ring in Fig. 2; $\omega=n \varphi+\beta_{0} \zeta$ denotes equivalent spiral angle of rotating ring; $\varphi$ denotes angle coordinate; $\beta_{0}=n \tan \alpha$ denotes the groove slope coefficient; $n$ denotes the number of spiral groove; $E$ denotes half depth of the groove; $\varepsilon$ denotes the iterative perturbation parameter; $A_{1}, A_{2}, B_{1}, B_{2}, c_{10}, c_{10}{ }^{\prime}, c_{11}, c_{11}{ }^{\prime}, c_{20}, c_{20}{ }^{\prime}, c_{21}, c_{21}{ }^{\prime}$ is integral constant and $\beta_{1}=n^{2}+\beta_{0}^{2}$ denotes the square sum of groove slope coefficient and spiral groove number; the parameters above are related to the rotating ring. $p$ denotes the medium pressure; $\Delta h$ denotes the axial gas film displacement.

Substituting Eq. (2) into Eq. (1) to get

$$
\begin{aligned}
& K=\frac{E p_{i}\left(\pi R_{o}^{2}-\pi R_{i}^{2}\right)}{(\delta+E)^{2}\left(1-\Delta h \cos \phi-\left(\frac{E \cos \omega}{E+\delta}\right)\right)^{2}} \times\left[\left(\eta_{1(\zeta)} \cos \omega \cos \phi_{\Delta} h+\eta_{2(\zeta)} \sin \omega \cos \omega \Delta h\right)-\left(\eta_{1(\zeta)} \cos \omega+\eta_{2(\zeta)} \sin \omega+\cos \omega\right)\right] \\
& +3\left(\pi R_{o}{ }^{2}-\pi R_{i}^{2}\right) p_{i} \beta_{0} \eta_{2(\zeta)}\left(\zeta_{0}-\zeta\right)^{2} E^{2}(\delta+E)^{-3}
\end{aligned}
$$

If the medium pressure and the parameters determined by the rotating ring design are not changed, it can be seen from Eq. (3) that the stability of gas film is only related to the axial gas film change. As the axial vibration of floating ring leads to the axial change of gas film, the axial change of gas film can be measured by the axial vibration of floating ring.

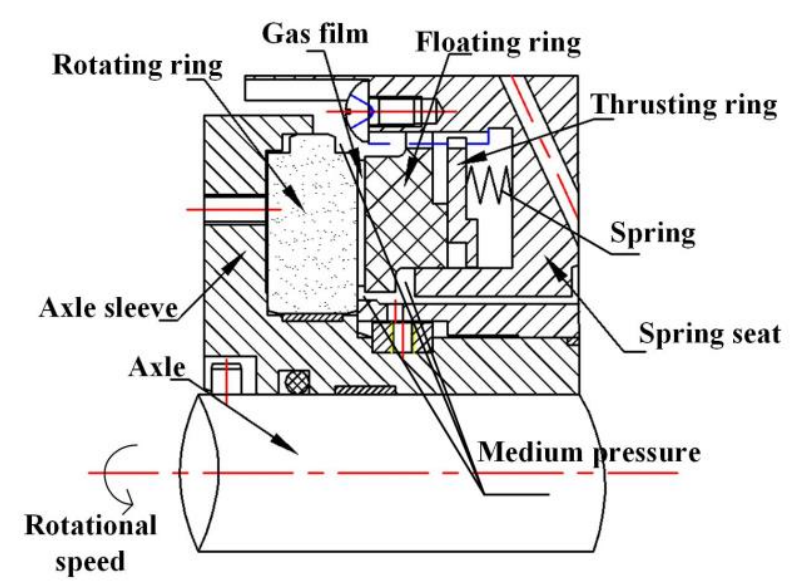

Fig. 1 The structure diagram of dry gas seal system 
In Fig. 1, the spring is in a state of compression between the fixed spring seat and the thrusting ring. Thus, the floating ring and thrusting ring are always moving together in the same direction. The FRS shown on the right side in Fig. 1 is simplified as a single degree of freedom system. The motion equation of FRS is [Yu et al., 2012]

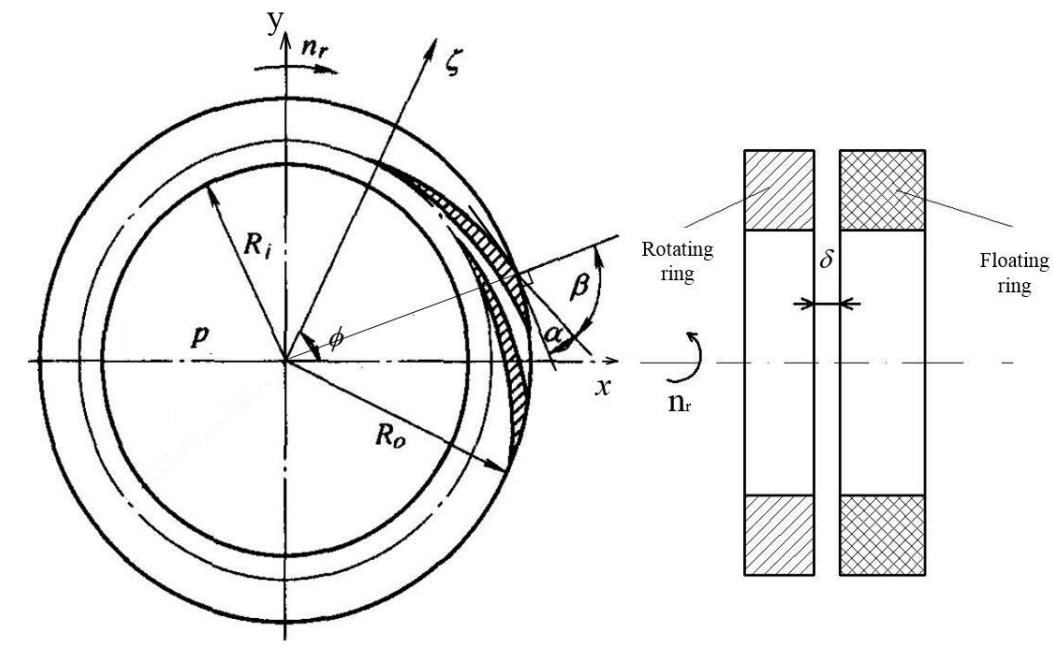

Fig. 2 The spiral groove parameters model and the gap of the sealing rings

$m \ddot{x}+c \dot{x}+k x=0$.

In a relatively short time the damping system can be ignored [Li et al., 2001]. Eq. (4) is simplified as

$m \ddot{x}+k x=0$.

Set $x=X_{m} \sin \psi t$ to get

$\left(k-m \psi^{2}\right) X_{m} \sin \psi t=0$.

Then

$k-m \psi^{2}=0$ or $\psi=\sqrt{k / m}$.

$m$ denotes the system mass; $c$ denotes the system damping; $k$ denotes the system stiffness; $x$ denotes the axial displacement; $t$ denotes time and $\psi$ denotes the system natural frequency.

Under the external excitation, the system equation of motion is

$$
\begin{aligned}
& m \ddot{x}+k x=F \sin w t, \\
& m a+k x=F \sin w t,
\end{aligned}
$$

$F$ is the exciting force from gas film; $w$ is the exciting frequency from rotational speed $n_{r}$.

Set $a=A \sin w t$ and $x=B \sin w t$, which is substituted into Eq. (9) to get

$m A \sin w t+k B \sin w t=F \sin w t$.

Substituting $A=-w^{2} B$ into Eq. (10) to get

$$
A=\frac{w^{2} F}{\left|w^{2} m-k\right|},
$$

$a$ denotes the axial acceleration.

The relationship between the axial change velocity of gas film and the axial vibration of FRS is established

$v_{\Delta h}=\int_{t 1}^{t 2} a d t$.

Under the unstable vibration of FRS, there is a possibility that the floating ring produces a violent axial vibration, 
which accelerates the axial displacement transient of gas film. The intense axial vibration of floating ring can lead to the drastic gas film change.

\section{Dynamic analysis and test experiment}

\subsection{Experiment design}

The vibration characteristics of the FRS were tested by the LMS Test Lab Measurement System. Furthermore, as the state of the gas film is reflected by the leakage directly, the leakage values were measured at the same time. The medium pressure and rotational speed are the most important operation conditions in the DGS. Considering the total-closed design of DGS device, the dynamic characteristics of FRS cannot be measured directly. The multi-stage discretization operation conditions (rated medium pressure $p$ and rated rotational speed $n_{r}$ reference to the nuclear reaction operation conditions of DGS device) were designed to test the vibration performance of the FRS.

ICP accelerometers with high sensitivity and small size (model: 333B30, sensitivity: $100 \mathrm{mV} / \mathrm{g}$, frequency range: $0.5 \mathrm{~Hz}-3 \mathrm{KHz}$, measurement range: $50 \mathrm{~g}$, weight: $4 \mathrm{~g}$ ) are adopted to ensure the data accuracy. ICP accelerometer is a kind of acceleration sensor embedded with micro IC amplifier. ICP is the engineering common name for such sensors. The ratio of the mass of accelerometers to the mass of parts measured is less than $10 \%$. The accelerometers were attached to the non-high pressure faces of the floating ring, thrusting ring and spring seat respectively, as shown in Fig. 3. The vibration signals were transmitted to the LMS data acquisition front through shielded cables. In the Signature signal analysis software module of LMS Test Lab, the sampling parameters were set according to the designed experiment requirements [Deng et al., 2015].

The leakage under the corresponding operation conditions mentioned above was tested by the high-precision leakage detection equipment. The detection accuracy is up to $0.001 \mathrm{~m}^{3} / \mathrm{h}$. During the test, the pressure inside the device was constant and the other accidental leakage caused by excessively high temperature was avoided.

The experiment design is shown in Table 1. The $p$ and $n_{r}$ control and the leakage detection is shown in Fig. 4.

Table 1. The vibration test experiment design of the FRS under different discrete working conditions

\begin{tabular}{c|c|c|c}
\hline \hline Experiment & Number & $n_{r}[\mathrm{r} / \mathrm{min}]$ & $p[\mathrm{MPa}]$ \\
\hline \multirow{5}{*}{$(1)$} & 1 & 6000 & 1 \\
& 2 & 6000 & 2 \\
& 3 & 6000 & 3 \\
& 4 & 6000 & 4 \\
& 5 & 6000 & 5 \\
& & & \\
& 6 & 3000 & 3 \\
& 7 & 4000 & 3 \\
& 8 & 5000 & 3 \\
& 9 & 6000 & 3 \\
& 10 & 7000 & 3 \\
\hline
\end{tabular}




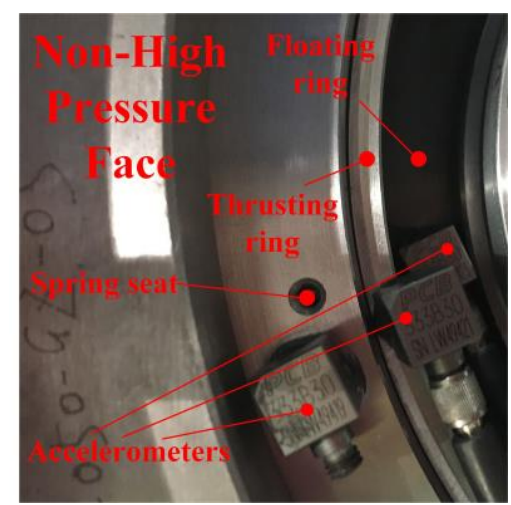

Fig.3 The accelerometers position affixed with the FRS and the non-high pressure face of the FRS

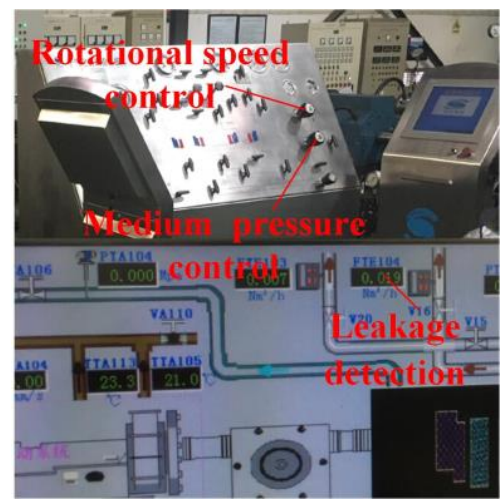

Fig.4 The $p$ and $n_{r}$ control and the leakage detection

\subsection{Experimental results}

The trend of acceleration response amplitude and leakage of the Experiment (1) for FRS is shown in Fig. 5. The amplitude response curve was in Fig. 5a and the leakage value curve is in Fig. 5b.

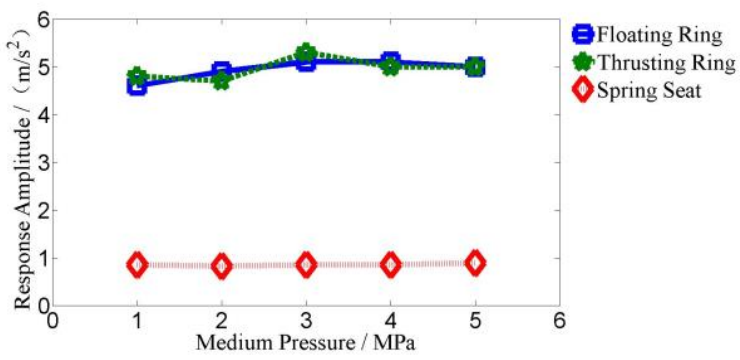

5(a) The floating ring (blue) vibration response amplitude, thrusting ring (green) vibration response amplitude and spring seat (red) vibration response amplitude under different medium pressure

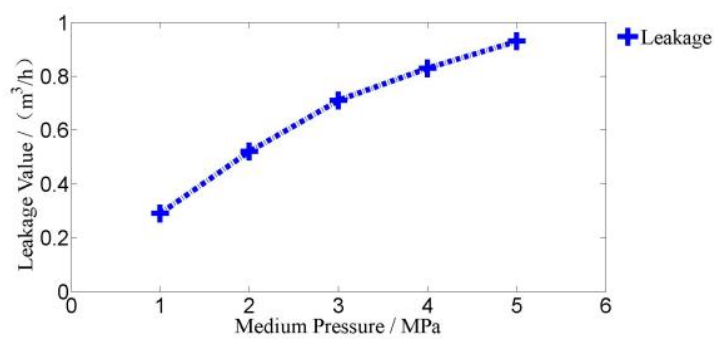

5(b) The leakage values under different medium pressure

Fig.5 The response curves of the Experiment (1) under different medium pressure

The trend of acceleration response amplitude and leakage of the Experiment (2) for FRS is shown in Fig. 6. The 
amplitude response curve is in Fig. 6a and the leakage value curve is in Fig. 6b.

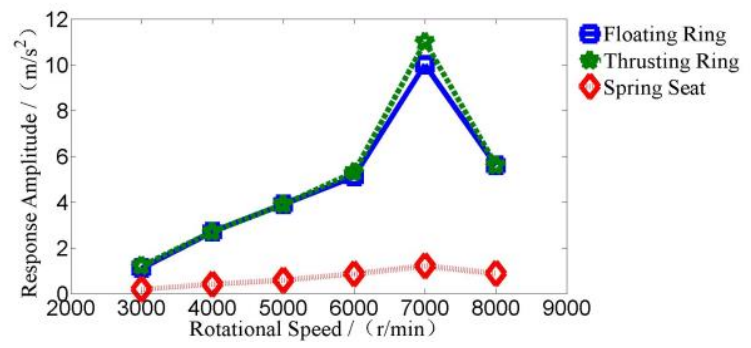

6(a) The floating ring (blue) vibration response amplitude, thrusting ring (green) vibration response amplitude and spring seat (red) vibration response amplitude under different rotational speed

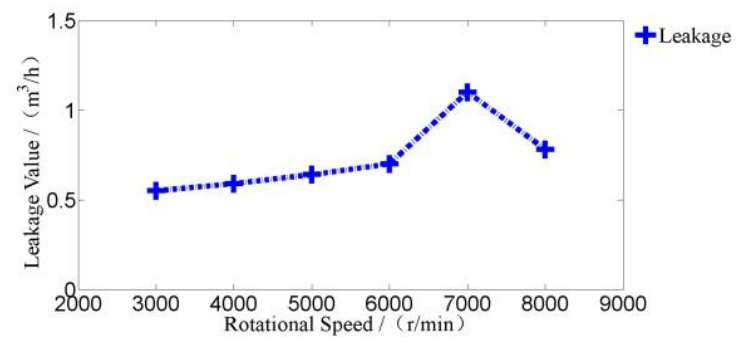

6(b) The leakage values under different rotational speed

Fig.6 The response curves of the Experiment (2) under different rotational speed

The result in Fig. 5a shows that the response amplitudes of the FRS do not change greatly under different medium pressures, which is consistent with the Eq. (11). The acceleration amplitude $A$ is independent of the medium pressure. However, under different rotational speed, the change of the acceleration amplitude of system shown in Fig. 6a is not monotonic. As the rotational speed increases, the amplitude rises first and then decreases, which is also consistent with the Eq. (11). When $w$ starts to increase, the value of $\left|w^{2} m-k\right|$ is gradually close to zero, and then $A$ increases. If $w$ continues to increase, the value of $\left|w^{2} m-k\right|$ will deviate from zero and increase, resulting in the decreases $A$. Fig. $5 \mathrm{~b}$ shows that with the increasing of the medium pressure, the amount of leakage increases gradually, which is the result of the design performance of seal itself and inevitable. The increasing rate of leakage gradually reduces as the medium pressure increases. It is due to the reason that the gas film stiffness will rise for certain extent with the medium pressure increasing in some extent. In Fig. 6b, with the initial increasing in rotational speed, the amount of leakage has increased a little. However, there is a leakage peak at $7000 \mathrm{r} / \mathrm{min}$. This is not a common problem for sealing leakage.

When the excitation frequency $w$ is close to the natural frequency $\psi$, the $A$ approaches to the peak leading to the axial change velocity of gas film $v_{\Delta h}$ too large. The resonance and excessive change velocity destroy the stability of gas film.

\section{The efficient integrated structure optimization}

To avoid the instability of gas film and the unreliability of DGS, an integrated structure optimization method based on the PSMA, RSM and PSO have been carried out to improve the dynamic property of FRS. The optimization framework is shown in Fig. 7. 


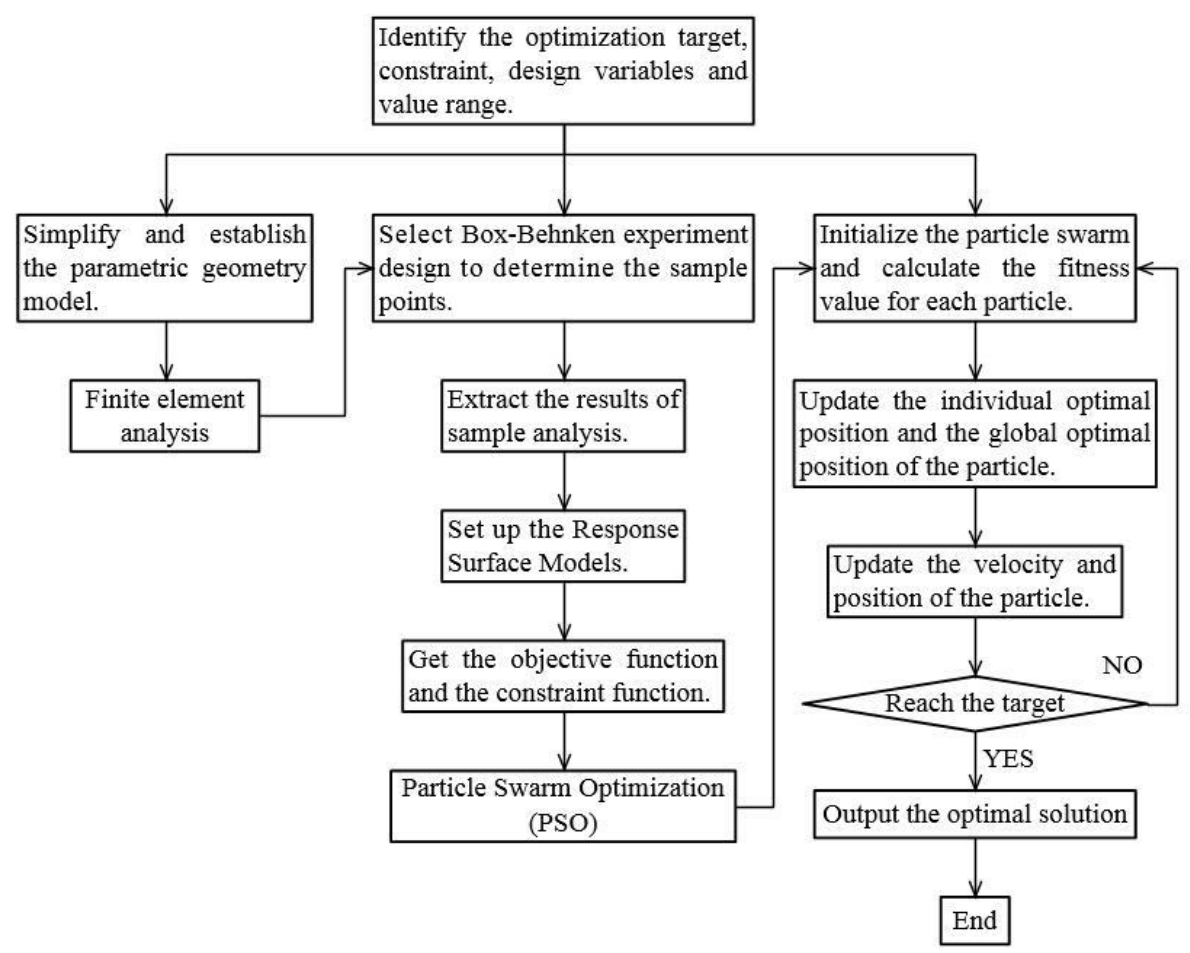

Fig.7 The integrated optimization design framework for structure optimization of FRS

(1) The natural frequency $f_{1}$ of the FRS is set as the objective function and the static deformation $f_{2}$ of the system is the constraint. Due to the special nested design of the FRS, the circumferential and radial dimension variables cannot be changed. The uniform spring stiffness $h_{1}$ and three main axial structure sizes including the thickness of the floating ring end $h_{2}$, the thickness of the thrusting ring tail $h_{3}$ and the thickness of the spring seat tail $h_{4}$ were chosen as the optimization variables, which, as shown in Fig.8. The value range of the 4 variables is

$$
\begin{gathered}
0.5 \mathrm{kN} / \mathrm{m} \leq h_{1} \leq 1.3 \mathrm{kN} / \mathrm{m} \\
0.8 \mathrm{~cm} \leq h_{2} \leq 1.1 \mathrm{~cm} \\
1.3 \mathrm{~cm} \leq h_{3} \leq 1.7 \mathrm{~cm} \\
0.35 \mathrm{~cm} \leq h_{4} \leq 0.5 \mathrm{~cm}
\end{gathered}
$$

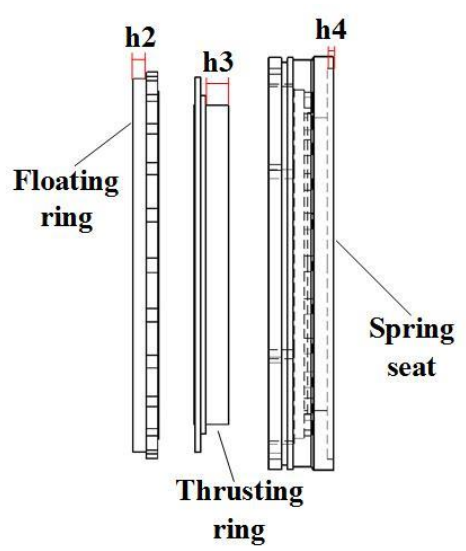

Fig.8 The optimization variables $h_{2} \quad h_{3}$ and $h_{4}$ of FRS 
(2) The parametric model of the FRS was established, and the PSMA based on finite element was accomplished through the ANSYS Workbench platform. The spectrum analysis of the axial vibration acceleration responses from the experiment proved the result of the PSMA by the peak picking method.

(3) The multiple groups PSMA of the sample points from the BB experiment design were carried out and the RS models of natural frequency and static deformation about the optimization variables were set up by the analysis data.

(4) With the alterable parameters in the FRS as optimization variables, the natural frequency as optimization target and the static deformation as constraint, the PSO was applied to find the optimal parameter value in the decision space $Q$.

The mathematical model of optimization design is as follows:

$$
\begin{array}{cc} 
& \min \left(-f_{1}\right) \\
\text { s.t. } & f_{2}(\vec{H}) \leq f_{2}^{\prime} \\
\vec{H}=\left(h_{1}, h_{2}, h_{3}, h_{4}\right)^{T} . \\
\underline{h}_{i} \leq h_{i} \leq \bar{h}_{i}(i=1,2,3,4)
\end{array} .
$$

Where $\vec{H}$ is the decision vector, $\vec{H} \in Q, \bar{h}_{i}$ is the upper limit of optimization variable, $\underline{h}_{i}$ is the lower limit of optimization variable, $f_{2}^{\prime}$ is the expected static deformation.

\subsection{Pre-Stressed Modal Analysis}

The Finite Element Model is one of the commonly used and mature optimization models, which is efficient and less loss [Lu and Xie, 2014]. According to the design requirements, the analysis model of the FRS was established. The assembly relationship of FRS is shown in Fig. 9. Reference to the contact requirements of the FRS, the contact relationship of floating ring and thrusting ring was set as 'Bonded'. The contact relationship of floating ring and spring seat was set as 'No Separation' and the contact relationship of thrusting ring and spring seat was set as 'No Separation' [Jiang et al., 2011.]. 'Bonded' and "No Separation" are the description about the contact type in ANSYS.

The boundary conditions of FRS according to the actual working conditions are shown in Fig. 10. The fixed constraints $\mathrm{C}$ was applied to the spring seat. A was the radial and axial medium pressure of the floating ring and thrusting ring. $\mathrm{B}$ was the gas film open force for the floating ring obtained based on the equilibrium condition of forces [Ding et al., 2008]. The 12 spring units were added between the spring seat and thrusting ring uniformly.

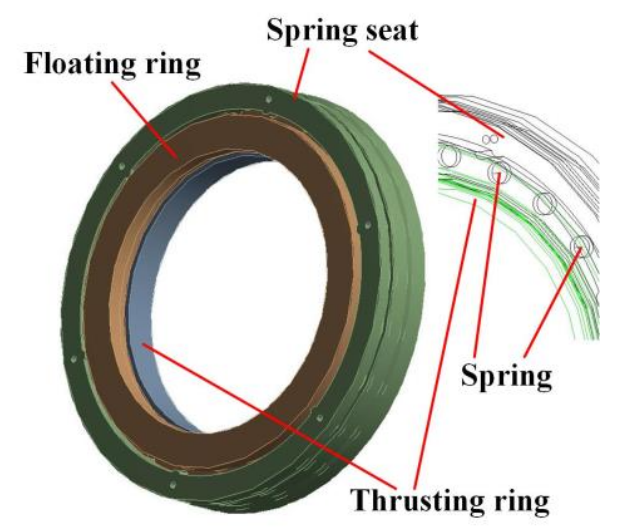

Fig.9 The assembly relationships of FRS (The floating ring is connected with the thrusting ring, which is embedded in the spring seat. The spring is between the spring seat and the thrusting ring.) 


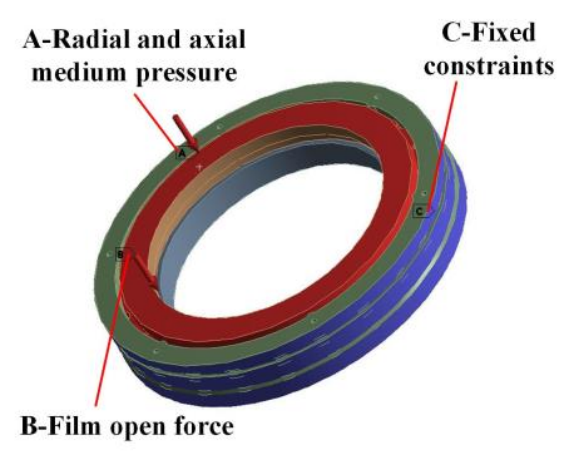

Fig.10 Boundary conditions of FRS (The fixed constraints $\mathrm{C}$ is on the spring seat. The radial and axial medium pressure $\mathrm{A}$ is on the floating ring and thrusting ring. The gas film open force B is on the high-pressure face of the floating ring.)

The structure of FRS bearing high rated medium pressure $p$ is likely to be affected. To get the natural frequencies $\psi$ and static deformations $\sigma$ that are consistent with the limit conditions, the PSMA for the FRS were carried out under the different rated medium pressure $p$. The result is shown in Table 2 . The structure of FRS is not affected by the rated rotational speed $n_{r}$, so the system natural frequencies keep constant under the different rated rotational speed.

Table 2. The natural frequencies and static deformations of FRS under different medium pressure

\begin{tabular}{c|c|c}
\hline \hline$p[\mathrm{MPa}]$ & $\psi[\mathrm{Hz}]$ & $\sigma[\mathrm{mm}]$ \\
\hline 1 & 118.9 & 5.7 \\
2 & 118.8 & 7.4 \\
3 & 118.6 & 8.6 \\
4 & 118.4 & 10 \\
5 & 118.3 & 11 \\
\hline
\end{tabular}

The first order mode shape of the FRS was axial vibration, in which the axial vibration value of floating ring and thrusting ring was large, and the spring seat was smaller, as shown in Fig. 11.

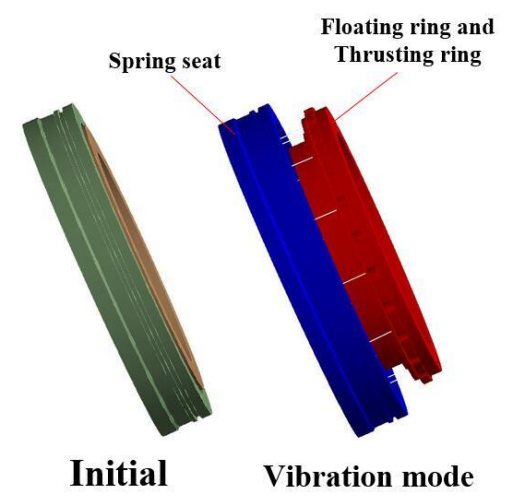

Fig.11 The first order mode shape of FRS

The spectrum analysis of the vibration acceleration response signals from the experiment was completed and the 
results are shown in Fig. 12. The peak picking method is simple and suitable for the non-dense modal identification. Ignoring excitation frequency, the abscissa corresponding to obvious same peaks between 0 and $500 \mathrm{~Hz}$ is the natural frequency of the system. Compared with the natural frequency results of model analysis, the error of experiment analysis results is small as shown in Table 3.

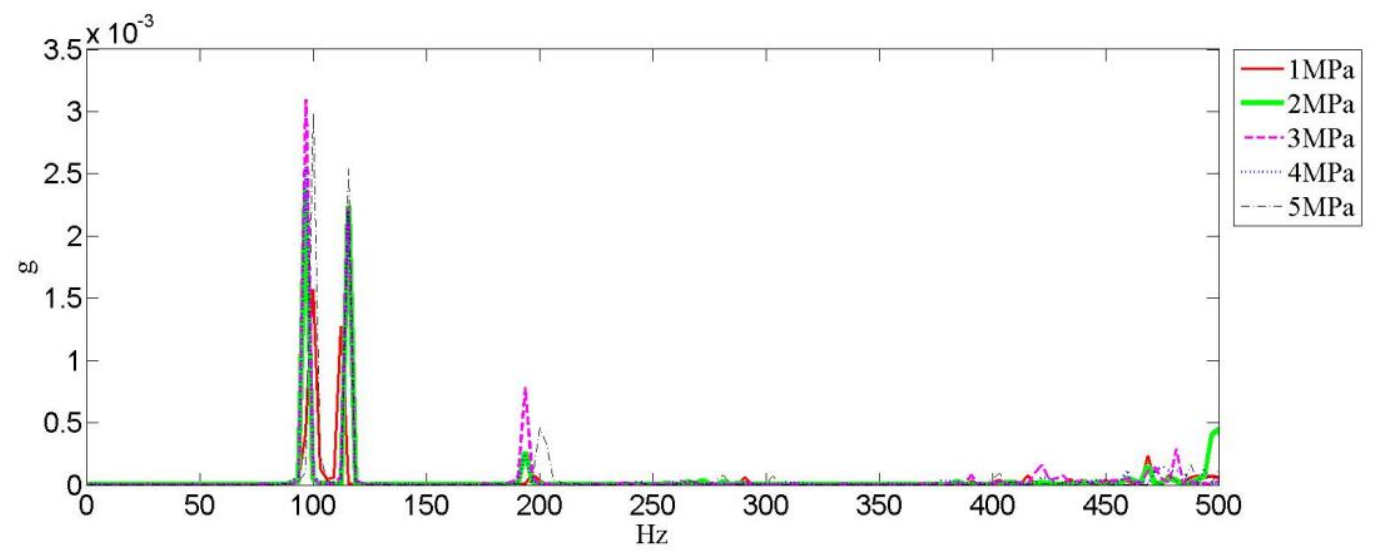

Fig.12 The spectrum analysis of signals under different medium pressures - 1MPa(red), 2Mpa(green), $3 \mathrm{MPa}$ (purple), $4 \mathrm{MPa}$ (blue) and 5MPa(black).

Table 3. Results of spectrum analysis and dynamic analysis of FRS

\begin{tabular}{c|c|c|c}
\hline \hline \multirow{2}{*}{$p[\mathrm{MPa}]$} & $\begin{array}{c}\text { Natural frequencies of } \\
\text { spectrum analysis [Hz] }\end{array}$ & $\begin{array}{c}\text { Natural frequencies of } \\
\text { dynamic analysis [Hz] }\end{array}$ & Error \\
\hline 1 & 116 & 118.9 & $2.4 \%$ \\
2 & 113 & 118.8 & $4.9 \%$ \\
3 & 116 & 118.6 & $2.2 \%$ \\
4 & 116 & 118.4 & $2.0 \%$ \\
5 & 116 & 118.3 & $1.9 \%$ \\
\hline
\end{tabular}

In Fig. 5a, the peak appears at the rotational speed of $7000 \mathrm{r} / \mathrm{min}$, which is consistent with the first order mode shape. Through the natural frequencies and first order mode shape, the correctness the pre-stressed modal analysis was verified. The excitation frequency $116.7 \mathrm{~Hz}$ is close to the system natural frequency $116 \mathrm{~Hz}$, namely $w \approx \sqrt{k / m}$. Resonance occurred and gas film instability appeared leading to serious leakage.

\subsection{The establishment of the natural frequency and static deformation RS models}

RSM [Myers et al., 1995] is a method of establishing the approximate functions between the target, the constraint, and the design variables through a reasonable experiment design. In practice, according to the engineering experience, the RS model should adopt a complete quadratic polynomial. The complete quadratic polynomial RS model of the 4 optimization variables is

$$
\begin{aligned}
& \tilde{f}=\alpha_{0}+\alpha_{1} h_{1}+\alpha_{2} h_{2}+\alpha_{3} h_{3}+\alpha_{4} h_{4}+ \\
& \alpha_{11} h_{1} h_{1}+\alpha_{22} h_{2} h_{2}+\alpha_{33} h_{3} h_{3}+\alpha_{44} h_{4} h_{4}+ \\
& \alpha_{12} h_{1} h_{2}+\alpha_{13} h_{1} h_{3}+\alpha_{14} h_{1} h_{4}+\alpha_{23} h_{2} h_{3}+\alpha_{24} h_{2} h_{4}+\alpha_{34} h_{3} h_{4}
\end{aligned}
$$

Let

$$
\left\{\begin{array}{l}
h_{0}=h_{0} \\
h_{1}=h_{1}, h_{2}=h_{2}, h_{3}=h_{3}, h_{4}=h_{4} \\
h_{5}=h_{1} h_{1}, h_{6}=h_{2} h_{2}, h_{7}=h_{3} h_{3}, h_{8}=h_{4} h_{4} \\
h_{9}=h_{1} h_{2}, h_{10}=h_{1} h_{3}, h_{11}=h_{1} h_{4}, h_{12}=h_{2} h_{3}, h_{13}=h_{2} h_{4}, h_{14}=h_{3} h_{4}
\end{array},\right.
$$




$$
\left\{\begin{array}{l}
\beta_{0}=\alpha_{0} \\
\beta_{1}=\alpha_{1}, \beta_{2}=\alpha_{2}, \beta_{3}=\alpha_{3}, \beta_{4}=\alpha_{4} \\
\beta_{5}=\alpha_{11}, \beta_{6}=\alpha_{22}, \beta_{7}=\alpha_{33}, \beta_{8}=\alpha_{44} \\
\beta_{9}=\alpha_{12}, \beta_{10}=\alpha_{13}, \beta_{11}=\alpha_{14}, \beta_{12}=\alpha_{23}, \beta_{13}=\alpha_{24}, \beta_{14}=\alpha_{34}
\end{array}\right.
$$

In Eq. (16) and Eq. (17), $H_{v}=\left(h_{1}, h_{2}, h_{3}, h_{4}\right)$ is the optimization variables. $\beta_{k}$ is the coefficients. The number of the coefficients is $k=(n+1)(n+2) / 2=15 . \quad \beta=\left(\beta_{0}, \beta_{1}, \cdots, \beta_{k-1}\right)^{T}$ and the least squares principle is used to determine the unknown coefficient. The number of the independent experiment design $\bar{m}$ should not be less than $k$, namely $\bar{m} \geq k$. Experiment design is to follow certain criteria to select the sample points, so that only a spot of points can make the approximate response function achieve high accuracy. With minimum test cycle, the Central Composite Design (CCD) and BB experiment design both can provide the complete information about experiment variables. $\bar{m}_{B}$ is the number of the Box-Behnken experiment design and $\bar{m}_{C}$ is the number of the Central Composite Design experiment design. Compared with the result of $\mathrm{CCD}$, the number of experiment design groups of $\mathrm{BB}$ is the same as $\mathrm{CCD}, \bar{m}_{B}=\bar{m}_{C}=25$. Because the values of sample points of BB experiment design are closer to the actual available value, the BB experiment design is selected to ensure the higher accuracy of the natural frequency and static deformation RS models.

\begin{tabular}{|c|c|c|c|c|c|c|c|c|c|}
\hline Number & $h_{1}$ & $h_{2}$ & $h_{3}$ & $h_{4}$ & Number & $h_{1}$ & $h_{2}$ & $h_{3}$ & $h_{4}$ \\
\hline 1 & 0 & 0 & 0 & 0 & 14 & 0 & -1 & -1 & 0 \\
\hline 2 & -1 & -1 & 0 & 0 & 15 & 0 & 1 & -1 & 0 \\
\hline 3 & 1 & -1 & 0 & 0 & 16 & 0 & -1 & 1 & 0 \\
\hline 4 & -1 & 1 & 0 & 0 & 17 & 0 & 1 & 1 & 0 \\
\hline 5 & 1 & 1 & 0 & 0 & 18 & -1 & 0 & -1 & 0 \\
\hline 6 & 0 & 0 & -1 & -1 & 19 & 1 & 0 & -1 & 0 \\
\hline 7 & 0 & 0 & 1 & -1 & 20 & -1 & 0 & 1 & 0 \\
\hline 8 & 0 & 0 & -1 & 1 & 21 & 1 & 0 & 1 & 0 \\
\hline 9 & 0 & 0 & 1 & 1 & 22 & 0 & -1 & 0 & -1 \\
\hline 10 & -1 & 0 & 0 & -1 & 23 & 0 & 1 & 0 & -1 \\
\hline 11 & 1 & 0 & 0 & -1 & 24 & 0 & -1 & 0 & 1 \\
\hline 12 & -1 & 0 & 0 & 1 & 25 & 0 & 1 & 0 & 1 \\
\hline 13 & 1 & 0 & 0 & 1 & & & & & \\
\hline
\end{tabular}
The result of BB experiment design is shown in Table 4 [Park and Nguyen, 2016].

Table 4. BB experiment design table 
$H=\left[\begin{array}{ccccc}1 & h_{1}^{(0)} & h_{2}^{(0)} & \ldots & h_{k-1}^{0} \\ 1 & h_{1}^{(1)} & h_{2}^{(1)} & \ldots & h_{k-1}^{1} \\ \vdots & \vdots & \vdots & & \vdots \\ 1 & h_{1}^{(\bar{m}-1)} & h_{2}^{(\bar{m}-1)} & \ldots & h_{k-1}^{(\bar{m}-1)}\end{array}\right]$ is the $m$ sample points. $f=\left\{\begin{array}{c}f^{(0)} \\ f^{(1)} \\ \vdots \\ f^{\bar{m}-1}\end{array}\right\}$ is the corresponding response values.

$\left\{\begin{array}{c}\tilde{f}^{(0)}=\sum_{i=0}^{k-1} \beta_{i} h_{i}^{(0)} \\ \tilde{f}^{(1)}=\sum_{i=0}^{k-1} \beta_{i} h_{i}^{(1)} \\ \vdots \\ \tilde{f}^{(\bar{m}-1)}=\sum_{i=0}^{k-1} \beta_{i} h_{i}^{\left({ }^{(}-1\right)}\end{array}\right.$

Let $S(\beta)=\sum_{j=0}^{\bar{m}-1}\left(\varepsilon^{(j)}\right)^{2}=\sum_{j=0}^{\bar{m}-1}\left(\sum_{i=0}^{k-1} \beta_{i} h_{i}^{(j)}-f^{(j)}\right)^{2} \rightarrow$ min . The necessary condition for the minimum

is $\frac{\partial S}{\partial \beta_{l}}=2 \sum_{j=0}^{\bar{m}-1}\left[h_{l}^{(j)}\left(\sum_{i=0}^{k-1} \beta_{i} h_{i}^{(j)}-f^{(j)}\right)\right]=0 \quad(l=0, \cdots, k-1) \cdot$ Namely, $(H \beta-f)^{T} H=0$

Then $\beta=\left(H^{T} H\right)^{-1} H^{T} f$.

After the approximate model is established, the prediction ability of the RS should be evaluated. The common evaluation indexes include $R^{2}, R_{a d j}^{2}$ and $\sigma_{R M S E}$.

$$
\begin{aligned}
& R^{2}=1-\frac{S S E}{S S Y} . \\
& R_{a d j}^{2}=1-\left(\frac{\bar{m}-1}{\bar{m}-k}\right) \frac{S S E}{S S Y} . \\
& \sigma_{R M S E}=\frac{1}{\bar{m} \bar{f}} \sqrt{\sum_{i=1}^{\bar{m}}\left(f_{i}-\tilde{f_{i}}\right)^{2}} . \\
& S S E=\sum_{i=1}^{\bar{m}}\left(f_{i}-\tilde{f_{i}}\right)^{2} \quad S S Y=\sum_{i=1}^{\bar{m}} f_{i}^{2}-\bar{m} \bar{f}^{2} .
\end{aligned}
$$

$R^{2}$ is the multiple correlation coefficients. The closer the $R^{2}$ to 1 is, the smaller the effect of error is. If $R^{2}=1$, it means that the regression equation can accurately describe the $f$. Namely, all of the observation points are on the surface determined by the regression equation.

$R^{2}$ describes the fitting degree of the RS and the value increases with the number of independent variables increasing in the regression equation. $R_{a d j}^{2}$ is the modified multiple correlation coefficient which takes the effect of the number of parameters $k$ into account. If the difference between $R^{2}$ and $R_{a d j}^{2}$ is large, the result is meaningless.

The closer the value of root mean square error $\sigma_{R M S E}$ to 0 is, the smaller the error between the response value and 
the response estimate is.

\subsection{The PSO of FRS}

PSO is an effective global optimization algorithm. Compared with the traditional evolutionary algorithm, PSO retains the global search strategy based on population. In addition, the velocity- position model is simple to operate and avoid the complex genetic operations. The unique memory in PSO can dynamically track the current search situation and adjust the search strategy accordingly. Since the solution in each generation has the double excellence of "self" learning and learning from "others", the optimal solution can be found in fewer iterations.

$H Z_{i}=\left(h z_{i 1}, h z_{i 2}, \cdots, h z_{i n}\right)$ is the current position of the particle $i . H V_{i}=\left(h v_{i 1}, h v_{i 2}, \cdots, h v_{i n}\right)$ is the current velocity of the particle $i$. pbest $t_{i}=\left(\right.$ pbest $_{i 1}$, pbest $_{i 2}, \cdots$, pbest $\left._{i n}\right)$ is the optimal position experienced by the particle $i$, named the individual optimal position.

$f_{1}(X)$ is the objective function that should be minimized, then the current optimal position of the particle $i$ is determined by

$$
\text { pbest }_{i}(t+1)=\left\{\begin{array}{l}
\operatorname{pbest}_{i}(t), f_{1}\left(H Z_{i}(t+1)\right) \geq f_{1}\left(\text { pbest }_{i}(t)\right) \\
H Z_{i}(t+1), f_{1}\left(H Z_{i}(t+1)\right)<f_{1}\left(\text { pbest }_{i}(t)\right)
\end{array} .\right.
$$

The number of particles in the population is set as $N$, and the best position gbest $(t)$ where all the particles in the population have experienced is the global optimal position.

$$
\operatorname{gbest}(t)=\min \left\{f_{1}\left(\text { pbest }_{1}(t)\right), \quad f_{1}\left(\text { pbest }_{2}(t)\right), \quad \cdots, \quad f_{1}\left(\text { pbest }_{N}(t)\right)\right\} .
$$

The velocity and position of the particle can be dynamically adjusted according to the flight experience of the individual and the population. The update equation of velocity and position is

$$
\begin{aligned}
& h v_{i j}(t+1)=w\left(h v_{i j}(t)\right)+c_{1} r_{1}\left(\text { pbest }_{i j}(t)-h z_{i j}(t)\right)+c_{2} r_{2}\left(\text { gbest }_{j}(t)-h z_{i j}(t)\right) . \\
& h z_{i j}(t+1)=h z_{i j}(t)+h v_{i j}(t+1)
\end{aligned}
$$

Where $i$ represents the particle $i$ and $j$ represents the $j$ th dimension of particle $i . h v_{i j}(t)$ is the $j$ th dimension velocity component of particle $i$ evolving to the $t$ th generation. $h z_{i j}(t)$ is the $j$ th dimension position component of particle $i$ evolving to the $t$ th generation. pbest $t_{i j}(t)$ represents the $j$ th dimension component of the individual optimal position pbest $t_{i}$ of particle $i$ evolving to the $t$ th generation. gbest $_{j}(t)$ represents the $j$ th dimension component of the optimal position gbest of the entire particles population evolving to the $t$ th generation. $w$ is the inertia weight. $c_{1}$ and $c_{2}$ is the acceleration factor. $r_{1}$ and $r_{2}$ is the random from 0 to 1 .

The complete quadratic RS models and the evaluation indexes are shown in Table 5, which demonstrates the accuracy of the RS models. The two RS models achieved the direct expressions of natural frequency and static deformation about the optimization variables including $h_{1}, h_{2}, h_{3}$ and $h_{4}$.

The optimization experiments show that the algorithm has better convergence performance as $w$ taking 0.7298 and $c_{1}=c_{2}=1.49618$ [Van Den Bergh et al., 2006, Eberhart et al., 2000]. The better search ability can be obtained by the large population size, leading to the slower search speed. Here the population size is $\mathrm{N}=40$. The maximum 
iteration number is $\mathrm{T}=100$. Based on the RS models in Table 5, the static deformation $f_{2}$ was set as less than $6 \times 10^{-2} \mathrm{~mm}$ following the design requirement as the constraint condition and the minimum negative value of the natural frequency $-f_{1}$ was set as target. PSO belongs to the global model and the velocity- position model avoid the complex operations. The global optimal particles provide information to other particles. The whole search update process was always following the current optimal solution, so the 4 variables $h_{1}, h_{2}, h_{3}$ and $h_{4}$ converged fast to the optimal solution. The 10 optimal solutions are shown in Table 6. The solutions of 10 groups are close, which indicate that the optimal solution is stable.

Table 5. Complete quadratic RS models and evaluation indexes of the $f_{1}$ and $f_{2}$

\begin{tabular}{|c|c|c|c|c|}
\hline Name & $\beta$ & $R^{2}$ & $R_{a d j}^{2}$ & $\sigma_{R M S E}$ \\
\hline $\begin{array}{l}\text { Function of } \\
\text { natural } \\
\text { frequency } f_{1}\end{array}$ & $\begin{array}{l}-6.827 e^{3}+11.9668 h_{1}+261.58 h_{2}+9.6938 e^{3} h_{3}-12.3666 h_{4} \\
-4.1777 h_{1}^{2}-88.2584 h_{2}^{2}-3406.69 e^{3} h_{3}^{2}-43.4328 h_{4}^{2} \\
-0.0796 h_{1} h_{2}-1.087 h_{1} h_{3}-0.1249 h_{1} h_{4} \\
-85.324 h_{2} h_{3}+14.7301 h_{2} h_{4} \\
+30.9857 h_{3} h_{4}\end{array}$ & 0.9907 & 0.9777 & 0.0028 \\
\hline $\begin{array}{l}\text { Function of } \\
\text { static } \\
\text { deformation } f_{2}\end{array}$ & $\begin{array}{l}0.0083+1.2555 e^{-5} h_{1}+3.934 e^{-4} h_{2}-0.0124 h_{3}+0.0017 h_{4} \\
-1.0211 e^{-5} h_{1}^{2}+1.9352 e^{-4} h_{2}^{2}+0.0047 h_{3}^{2}+1.8341 e^{-4} h_{4}{ }^{2} \\
-6.0654 e^{-8} h_{1} h_{2}+6.5927 e^{-6} h_{1} h_{3}+2.8904 e^{-7} h_{1} h_{4} \\
-5.1342 e^{-4} h_{2} h_{3}-1.2386 e^{-5} h_{2} h_{4} \\
-0.0013 h_{3} h_{4}\end{array}$ & 0.9999 & 0.9999 & $4.6115 e^{-8}$ \\
\hline
\end{tabular}

Table 6. The 10 groups optimized solutions of FRS by PSO

\begin{tabular}{c|c|c|c|c|c}
\hline \hline Number & $f_{1}[\mathrm{~Hz}]$ & $h_{1}[\mathrm{kN} / \mathrm{m}]$ & $h_{2}[\mathrm{~cm}]$ & $h_{3}[\mathrm{~cm}]$ & $h_{4}[\mathrm{~cm}]$ \\
\hline 1 & 142.08 & 1.2391 & 0.8332 & 1.4142 & 0.4498 \\
2 & 142.0813 & 1.2372 & 0.8359 & 1.4141 & 0.45 \\
3 & 142.0813 & 1.2329 & 0.8353 & 1.4141 & 0.45 \\
4 & 142.0811 & 1.2387 & 0.8368 & 1.4141 & 0.45 \\
5 & 142.0803 & 1.2459 & 0.8353 & 1.4145 & 0.45 \\
6 & 142.0805 & 1.2343 & 0.8385 & 1.4142 & 0.45 \\
7 & 142.0812 & 1.2282 & 0.8355 & 1.4141 & 0.45 \\
8 & 142.0803 & 1.2491 & 0.8358 & 1.4141 & 0.45 \\
9 & 142.0798 & 1.2149 & 0.8351 & 1.4143 & 0.45 \\
10 & 142.0805 & 1.2420 & 0.8330 & 1.4143 & 0.45 \\
\hline
\end{tabular}


The optimized design variables of system is shown in Table 7. The optimized natural frequency reaches above $142.1 \mathrm{~Hz}$ more than $133 \mathrm{~Hz}(8000 \mathrm{r} / \mathrm{min})$, which greater than the range of $n$.

From Table 6 and 7, the spring stiffness is proportional to the natural frequency. However, the thickness of the floating ring end and the thickness of the thrusting ring tail are inversely proportional to the natural frequency, among them $h_{2}$ has greater sensitivity. From the comparison of initial design variables and optimized design variables, the variables values must be modified to improve the FRS natural frequency. The spring stiffness $h_{1}$ should be increased.

The thickness of the floating ring end $h_{2}$ and the thickness of the thrusting ring tail $h_{3}$ should be reduced accordingly.

The thickness of the spring seat tail has little effect on the natural frequency. $h_{4}$ should be unchanged. As the variables $h_{1} \quad h_{2} \quad h_{3}$ and $h_{4}$ possess the engineering actual significance, their values should be rounded. Then $h_{1}$ $h_{2} \quad h_{3}$ are determined as $1.2 \mathrm{kN} / \mathrm{m} 0.8 \mathrm{~cm} 1.4 \mathrm{~cm}$ respectively and $h_{4}$ still is $0.45 \mathrm{~cm}$. The optimized FRS natural frequency is 140.7 increased by $15 \%$.

Table 7. Comparison of the initial and optimized results of FRS variables $h_{1}, h_{2}, h_{3}$ and $h_{4}$

\begin{tabular}{c|c|c|c}
\hline \hline Variables & initial & Optimized \\
design variables & design variables & Variables \\
rounding
\end{tabular}

\section{Conclusion}

Based on the relationship between the gas film stability and the vibration characteristics of FRS, it is obvious that the dynamic characteristics of FRS is related to the reliability of the DGS with precision sealing requirements. The test experiment about the vibration acceleration and leakage of the FRS was set up under the multi-stage discretization operation conditions. The trend of the axial vibration acceleration response amplitudes is consistent with the Eq. (11) under different operation conditions. The trend of the vibration acceleration and leakage meets the Eq. (12). The leakage peak at $7000 \mathrm{r} / \mathrm{min}$ is not a common problem. The peak demonstrates the system resonance and the instability of gas film. To obtain the absolute reliability of DGS system, the structure of the FRS should be optimized improve the dynamic property. PSMA achieved the system natural frequency and static deformation simultaneously. The accuracy of PSMA was confirmed by the spectrum analysis and vibration acceleration response. Using RSM, two direct expressions about the natural frequency and the static deformation of the optimization variables were obtained. Taking the natural frequency as the objective function and the static deformation as the constraint condition, the system efficient optimization was completed through the PSO. The natural frequency of the optimized FRS is increased by 
$15 \%$ reaching above $140 \mathrm{~Hz}$, which is higher than the $8000 \mathrm{r} / \mathrm{min}$. The influence degree of the optimization variables on the system natural frequency was also presented. The dynamic analysis of FRS supplements the dynamic characteristics study of the whole DGS system. By means of PSMA, BB experiment design, RSM and PSO, the parameter integrated optimization of DGS system applied to nuclear reaction sealing was realized. According to the optimization result, a size design basis was provided for product designers. The prediction and optimization of system operating modal is the future work.

\section{Acknowledgements}

The authors wish to express sincere thanks to supports by the National Key Technology R\&D Program of China [No.2015BAF27B01], National Science and Technology Support Project [No. 2017YFB1401702-2] and Science and Technology Support Plan Project of Sichuan province [No. 2017GZ0061]

\section{References}

Deng, C. Y., Heng, L. and Yin, Q., Application of bolt joints dynamic parameters identification in machine tools based on partially measured frequency response functions, Journal of Vibroengineering, Vol. 17, No. 3 (2015), pp. 1090-1104.

Ding, X. X., The research on steady - state and dynamical characterization of hubricating gaseous film in the sprial grooved gas seals, Lanzhou University of Technology (in Chinese), Lanzhou, China, (2008).

Ding, X. X., Pu, J. J. and Han, M. J., Calculation and analysis of gas film stiffness in the spiral groove gas seal based on the second order slip boundary, Journal of Mechanical Engineering (in Chinese), Vol. 47, No. 23 (2011), pp. 119-124.

Eberhart, R. C. and Shi, Y., Comparing inertia weights and constriction factors in particle swarm optimization, Proceedings of the IEEE Congress on Evolutionary Computation, Piscataway, (2000).

Faria, M. T. C., An efficient finite element procedure for analysis of high-speed spiral groove gas face seals, Journal of Tribology, Vol. 123, No. 1 (2001), pp. 205-210.

Jiang, H., Guan, Y. S. and Qiu, Z. C., Dynamic and static multi-objective optimization of a vertical machining center based on response surface method, Journal of Mechanical Engineering (in Chinese), Vol. 47, No. 11 (2011), pp. 125-133.

Jiao, A. Y., Quan, H. J. and Li Z. Z., Study of magnetic abrasive finishing in seal ring groove surface operations, The International Journal of Advanced Manufacturing Technology, No. 85 (2016), pp. 1195-1205.

Kittidecha, C. and Marasinghe, A. C., Application of Kansei Engineering and Box-Behnken response surface methodology for shape parameter design: A case study of wine glass, Journal of Advanced Mechanical Design, Systems, and Manufacturing, Vol. 9, No. 5 (2015), pp. 1-12.

Lee, S. C. and Zheng, X. L., Analyses of both steady behavior and dynamic tracking of non-contacting spiral-grooved gas face seals, Computers and Fluids, No. 88 (2013), pp. 46-55.

Li, D. B. and Lu, Q. H., Experimental Modal Analysis and Application, Science Press (in Chinese), Beijing, China, (2001).

Li, W., Yang, Y. and Sheng, D. R., Nonlinear dynamic analysis of a rotor/bearing/seal system, Journal of Zhejiang University-Science A, Vol. 12, No. 1 (2011), pp. 408-416.

Lu, X. H. and Xie, X. P., Multi-objective structural optimization of hub unit bearing using response surface methodology and genetic algorithm, Journal of Advanced Mechanical Design, Systems, and Manufacturing, Vol. 8, No. 3 (2014), pp. 1-10.

Miller, B. and Green, I., Numerical Techniques for Computing Rotor Dynamic Properties of Mechanical Gas Face Seals, Journal of Tribology, Vol. 124, No. 4 (2002), pp. 755-761.

Myers, R. H. and Montgometry, D. C., Response Surface Methodology, Wiley and Sons, New York, 1995.

Park, H. S. and Nguyen, T.T., Multi-objective optimization of turning process for hardened material based on hybrid approach, Journal of Advanced Mechanical Design, Systems, and Manufacturing, Vol. 10, No. 8 (2016), pp. 1-14.

Sui, Y. K. and Yu, H. P., Improvement of Response Surface Methodology and Application to Engineering Optimization, Science Press (in Chinese), Beijing, China, (2010).

Tan, F., Yin, Q. and Dong, G. H., An optimal convective heat transfer coefficient calculation method in thermal analysis of spindle system, The International Journal of Advanced Manufacturing Technology, No. 91 (2017), pp. 2549-2560. 
Van Den Bergh, F. and Engelbrecht, A. P., A study of particle swarm optimization particle trajectories, Information Sciences, Vol. 176, No. 8 (2006), pp. 937-971.

Wang, X. Y., Meng, X. K. and Liu, X., Coupling model and performance analysis of hydrodynamic mechanical seal for reactor coolant pump, Tribology, Vol. 33, No. 2 (2013), pp. 169-177.

Wang, Y. M., Huang, W. F. and Li, Y.J., Mechanical seals for primary circuit of nuclear power plants, Tribology, Vol. 31, No. 4 (2011), pp. 408-416.

Xie, Z. C., Steve Shepard Jr, W. and Woodbury, K. A., Design optimization for vibration reduction of viscoelastic damped structures using genetic algorithms, Shock and Vibration, No. 16 (2009), pp. 455-466.

Yu, S. R., Zhu, L. and Ding, X. X., Nonlinear stability analysis on axial vibration of dry gas seals system with gas gas film and seal rings, Journal of Vibration and Shock (in Chinese), Vol. 31, No. 15 (2012), pp. 101-104.

Zhou, J. F. and Gu, B. Q., Influence of micro perturbation on performance of liquid spiral groove mechanical seal, Tribology (in Chinese), Vol. 28, No. 4 (2008), pp. 327-332. 